\section{A Set of Monte Carlo Subroutines for} Treating the Physics of Compton Scattering

$$
\begin{gathered}
\text { 2ECEI VED } \\
\text { DEC } 16^{1998} \\
\text { OSTI }
\end{gathered}
$$

\section{Los Alamos \\ NATIONAL LABORATORY}

Los Alamos National Laboratory is operated by the University of California for the United States Department of Energy under contract W-7405-ENG-36. 
Edited by Jeff Skiby, Group CIC-1

Prepared by Karen Griggs, Group NIS-5

An Affirmative Action/Equal Opportunity Employer

This report was prepared as an account of work sponsored by an agency of the United States Government. Neither The Regents of the University of California, the United States

Government nor any agency thereof, nor any of their employees, makes any warranty, express or implied, or assumes any legal liability or responsibility for the accuracy, completeness, or usefulness of any information, apparatus, product, or process disclosed, or represents that its use would not infringe privately oumed rights. Reference herein to any specific commercial product, process, or service by trade name, trademark, manufacturer, or otherwise, does not necessarily constitute or imply its endorsement, recommendation, or farooring by The Regents of the Unizersity of California, the United States Government, or any agency thereof. The views and opinions of authors expressed herein do not necessarily state or reflect those of The Regents of the University of California, the United States Godernment, or any agency thereof. Los Alamos National Laboratory strongly supports academic freedom and a researcher's right to publish; as an institution, however, the Laboratory does not endorse the viezopoint of a publication or guarantee its technical correctness. 


\section{DISCLAIMER}

Portions of this document may be illegible in electronic image products. Images are produced from the best available original document. 
A Set of Monte Carlo Subroutines for

Treating the Physics of Compton Scattering

Jonathan Earnhart

John Lestone

Thomas Prettyman 
0

0

0

0

0

-

- 


\section{CONTENTS}

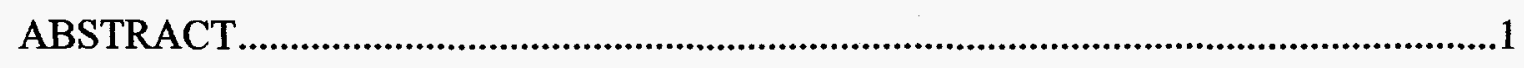

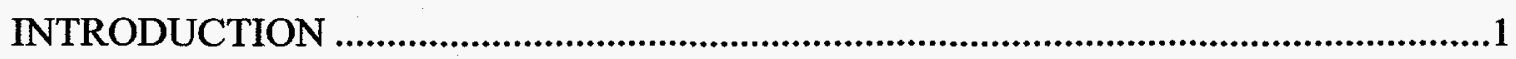

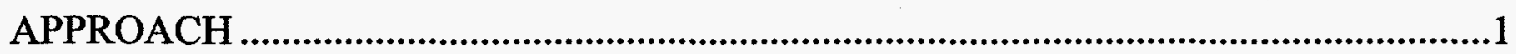

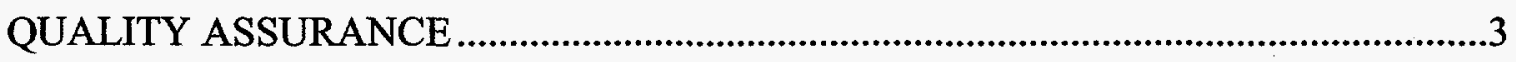

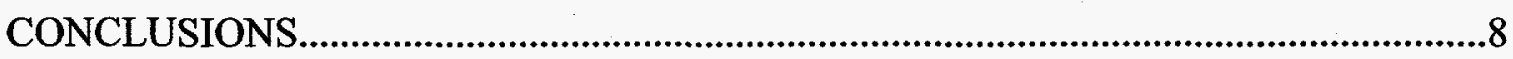

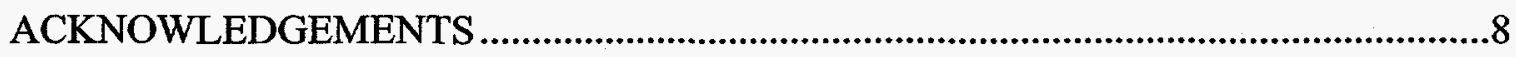

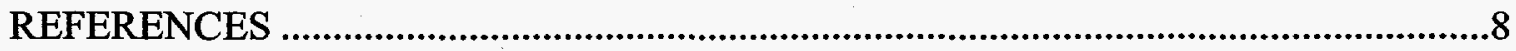

APPENDIX A - Incoherent Scattering Function Rejection Technique...................................9

APPENDIX B - Users' Manual ........................................................................................10

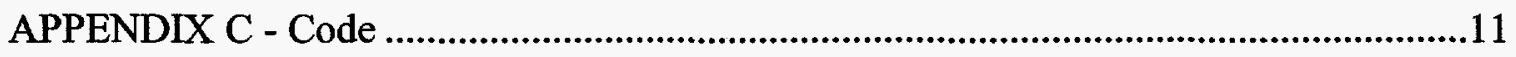




\title{
A SET OF MONTE CARLO SUBROUTINES FOR TREATING THE PHYSICS OF COMPTON SCATTERING
}

\author{
by
}

Jonathan Earnhart, John Lestone, and Tom Prettyman

\begin{abstract}
A set of portable Monte Carlo subroutines is presented to treat the physics of Compton scattering. Electron binding energies are included by the modification of the Klein-Nishina probability distribution by the incoherent scattering function. In addition, the scattered photon's energy is calculated by taking into account the momentum distribution of the electron. These subroutines have been verified and validated by calculating the total cross section over incident photon energies of $10 \mathrm{keV}$ to $100 \mathrm{MeV}$ for elements of $Z=1$ to $Z=100$ and are within $1.08 \%$ of published values.
\end{abstract}

\section{INTRODUCTION}

Compton scattering of photons off of electrons has been described by the Klein-Nishina formula. This treatment assumes the photon interacts with a free unbound electron. In order to account for the effect of the binding energies between the electrons and the nucleus on total atom Compton scattering, a factor for incoherent scattering is introduced. The final energy of the scattered photon depends on the electron's momentum distribution in its particular subshell around the atom. This distributes the scattered photon's energy about the energy predicted by the Compton energy-angle relation. A consistent approach, which includes the physics of electrons in bound states, was implemented into two simple Monte Carlo subroutines. The presented techniques assume that no energy is absorbed by the atoms during the scattering process and the atoms are free, i.e., no molecular effects. These routines were verified and validated by comparisons of the double differential, differential, and total incoherent scattering cross sections to values published in literature.

\section{APPROACH}

Once a Compton event is indicated in the simulation, the parameters governing the interaction are initial photon energy and atomic number of the atom involved in the scattering process. If the medium in which a Compton event occurs is a compound, the specific element the photon interacts with is sampled based on the fraction of electrons associated with each element in the compound. The code provides output of the final scattered photon energy and cosine of the polar scattering angle. Because only unpolarized photons are considered, the azimuthal scattering angle is sampled uniformly over the range $[0,2 \pi]$. 
The cosine of the scattering polar angle, $\mu$, is calculated using the Kahn rejection technique which samples from the Klein-Nishina formula exactly [1]. Another rejection technique is used to account for the incoherent scattering function, $S\left(x\left(\mu, \alpha_{i}\right), Z\right)$, where the scattered polar angle is retained with a probability of

$$
\frac{S\left(x\left(\mu, \alpha_{i}\right), Z\right)}{S\left(x\left(-1, \alpha_{i}\right), Z\right)} \leq 1 .
$$

A description of this technique is contained in Appendix A. The incoherent scattering function is tabulated as a function of the momentum transfer variable, $x\left(\mu, \alpha_{i}\right)$, in units of inverse Angstroms,

$$
x\left(\mu, \alpha_{i}\right)=29.143 \alpha_{i} \sqrt{1-\mu},
$$

where $\alpha_{i}$ is the initial photon energy in units of the rest energy of an electron and $m_{0} c^{2}=511 \mathrm{keV}$ [2]. Log-log interpolation is used to sample the values of the incoherent scattering function between the tabulated points, except between the first two points where linear interpolation is used.

Once the scattering polar angle is determined, the scattered photon's energy is calculated using shellwise Compton profiles as probability distribution functions. The Compton profiles are tabulated as a function of the projection of the electron precollision momentum on the momentum transfer vector of the photon. This projected momentum is described by

$$
p_{z}=-137 \frac{E_{i}-E_{s}-E_{i} E_{s}(1-\mu) / m_{0} c^{2}}{\sqrt{E_{i}^{2}+E_{s}^{2}-2 E_{i} E_{s} \mu}},
$$

where $p_{z}$ is the momentum in atomic units of $m_{0} e^{2} / \hbar$ (the average electron momentum in the ground state of hydrogen) and $E_{s}$ is the scattered photon energy [3]. The Compton profiles, $J_{k}\left(p_{z}, Z\right)$, for the elements $1 \leq Z \leq 102$ have been calculated and are symmetric about $p_{x}=0$ [4]. Note that these profiles are related to the incoherent scattering function by

$$
S\left(\mu, \alpha_{i}, Z\right)=\sum_{k} \int_{-\infty}^{p_{k, \max }} J_{k}\left(p_{z}, Z\right) d p_{z}
$$

where the subscript $\mathrm{k}$ refers to the particular electron subshell and $p_{k, \max }$ is obtained from the equation for $p_{z}$ by substituting $E_{s}=E_{i}-E_{k}^{b}$ ( $E_{k}^{b}$ is the binding energy of the electrons in the $\mathrm{k}^{\text {th }}$ shell).

The procedure begins by sampling the particular subshell of the electron that interacts with the photon. This is done based on the number of electrons in each subshell. A value of $p_{z}$ is sampled from a probability distribution function based on the Compton profile, 


$$
\xi=\frac{\int_{0}^{p_{z}} J_{k}\left(p_{z}^{\prime}\right) d p_{z}^{\prime}}{\int_{0}^{100} J_{k}\left(p_{z}^{\prime}\right) d p_{z}^{\prime}},
$$

where $\xi$ is a random number from uniform distribution contained on $[0,1]$. These integrals are calculated numerically assuming linear interpolation between the points (the denominator is precomputed). Once the value of $p_{z}$ is sampled, the scattered photon energy is the only unknown in the momentum equation. The equation is quadratic in $E_{s}$,

where

$$
A E_{s}^{2}+B E_{s}+C=0,
$$

$$
\begin{aligned}
& A=\left(\frac{p_{z}}{137}\right)^{2}-\left(\frac{E_{i}(1-\mu)}{m_{0} c^{2}}\right)^{2}-\frac{2 E_{i}(1-\mu)}{m_{0} c^{2}}-1, \\
& B=-2\left(\frac{p_{z}}{137}\right)^{2} E_{i} \mu+2 \frac{E_{i}^{2}(1-\mu)}{m_{0} c^{2}}+2 E_{i}, \text { and } \\
& C=\left(\frac{p_{z}}{137} E_{i}\right)^{2}-E_{i}^{2} .
\end{aligned}
$$

Of the two possible roots, one is randomly selected. If the scattered energy is greater than the maximum possible value determined by $E_{s, \max }=E_{i}-E_{b}$ ( $E_{b}$ is the binding energy of an electron in the $\mathrm{k}^{\text {th }}$ subshell [5]), or is negative, the sample is rejected and the process is repeated. Occasionally, imaginary roots are possible due to numerical fluctuations. When this occurs, the sample is rejected and the process is repeated. This technique was used in the EGS4 Monte Carlo code for including the Doppler broadening of a Comptonscattered photon's energy [6].

\section{QUALITY ASSURANCE}

The output of the Doppler broadening subroutine (doppler.for) has been used to calculate the profile of the double differential Compton scattering cross section, at a given initial energy and scattering angle, as a function of scattered energy. These results are compared with theoretical calculations of the relativistic impulse approximation of Ribberfors using Dirac-Hartree-Fock-Slater radial wave functions [7]. Specific comparisons are presented in the following figures (Figs. 1-4) for scattering off of lead and carbon, at energies of 344 and $1408 \mathrm{keV}$, and scattering angles of 5 and 15 degrees. 

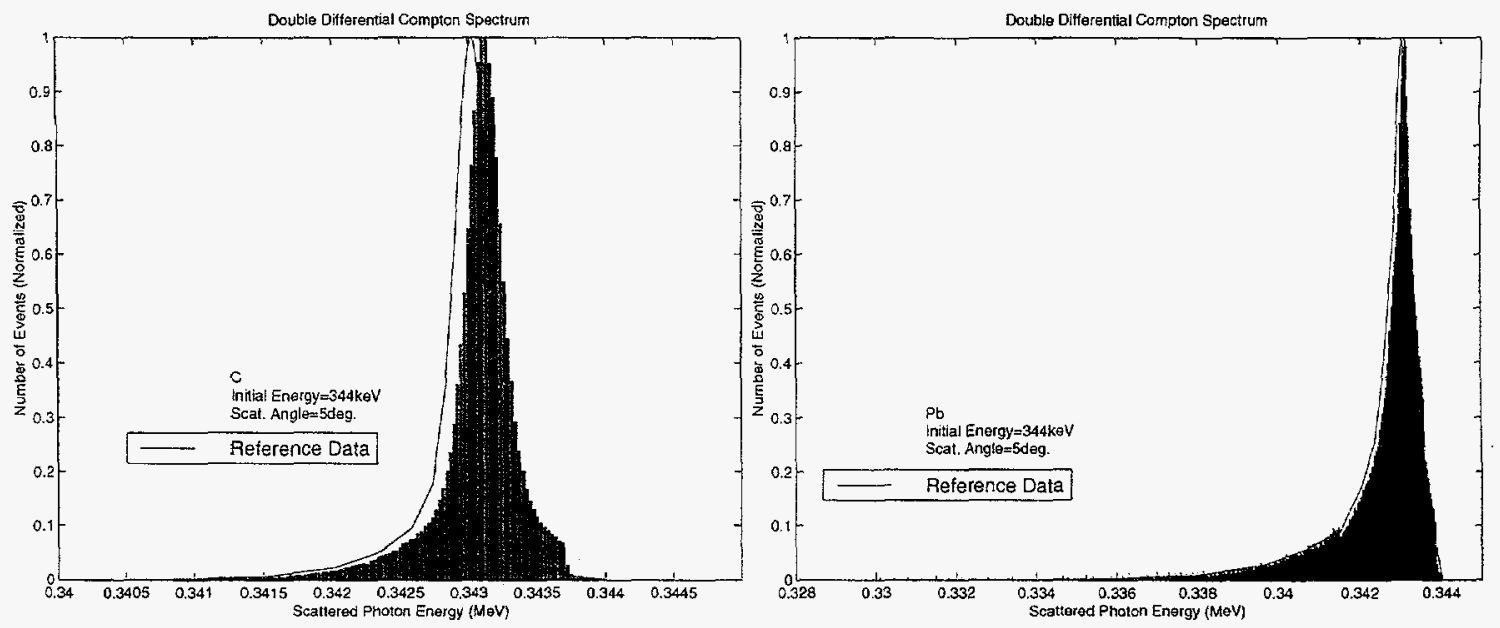

Fig. l. Comparison of Monte Carlo simulation results (histogram) and theoretical calculations (red line) of the double differential Compton scattering profiles for carbon (left) and lead (right) for an initial photon energy of $344 \mathrm{keV}$ and a scattering angle of 5 degrees.
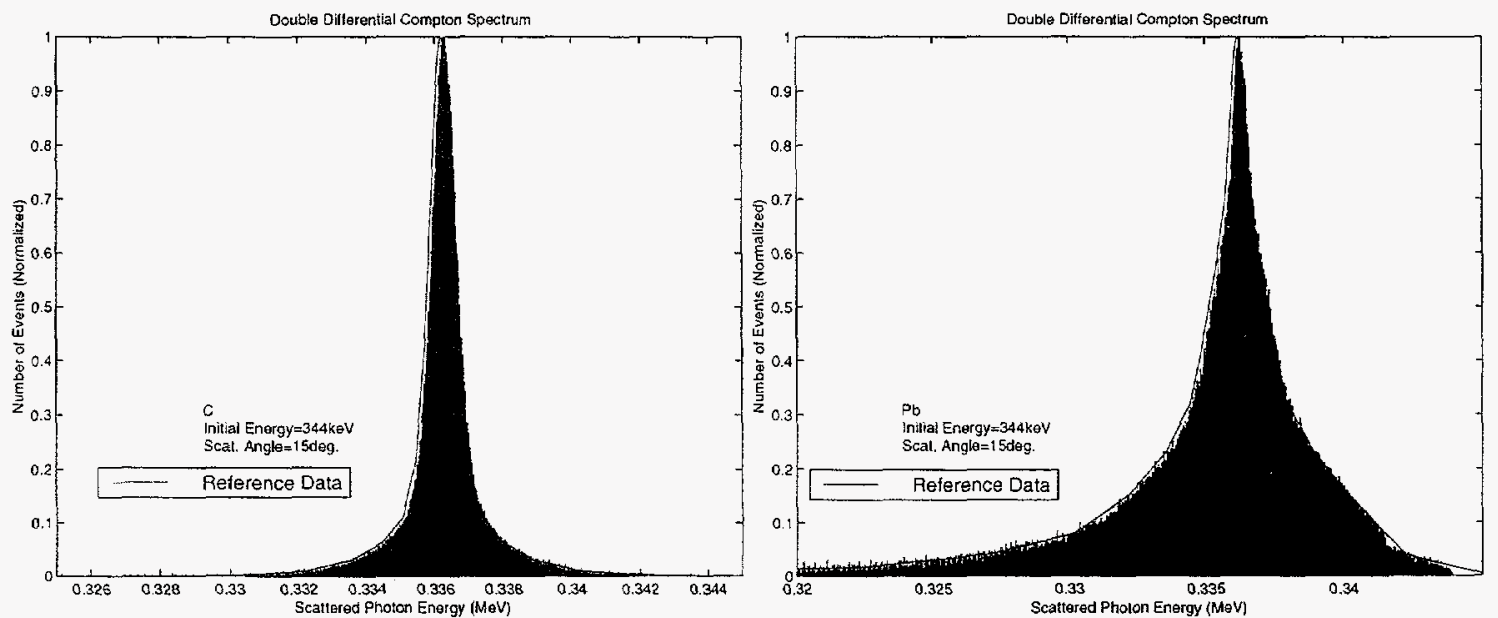

Fig. 2. Comparison of Monte Carlo simulation results (histogram) and theoretical calculations (red line) of the double differential Compton scattering profiles for carbon (left) and lead (right) for an initial photon energy of $344 \mathrm{keV}$ and a scattering angle of 15 degrees. 

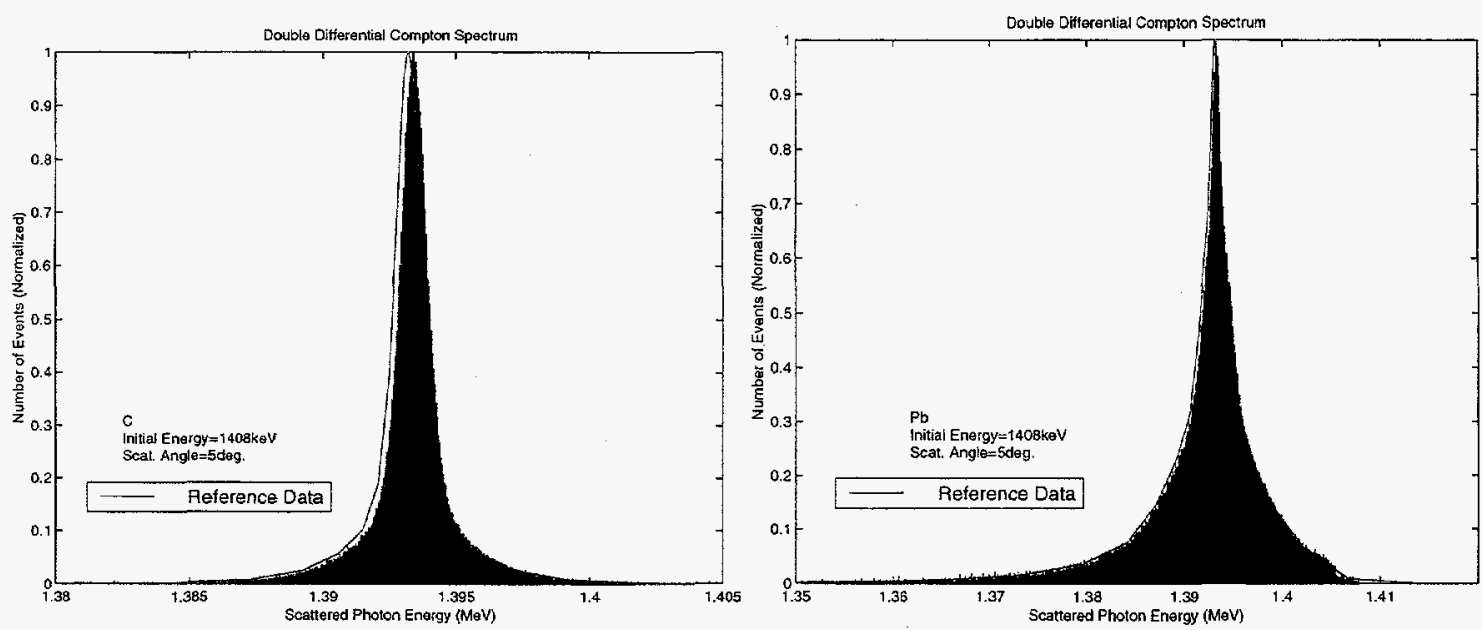

Fig. 3. Comparison of Monte Carlo simulation results (histogram) and theoretical calculations (red line) of the double differential Compton scattering profiles for carbon (left) and lead (right) for an initial photon energy of $1408 \mathrm{keV}$ and a scattering angle of 5 degrees.
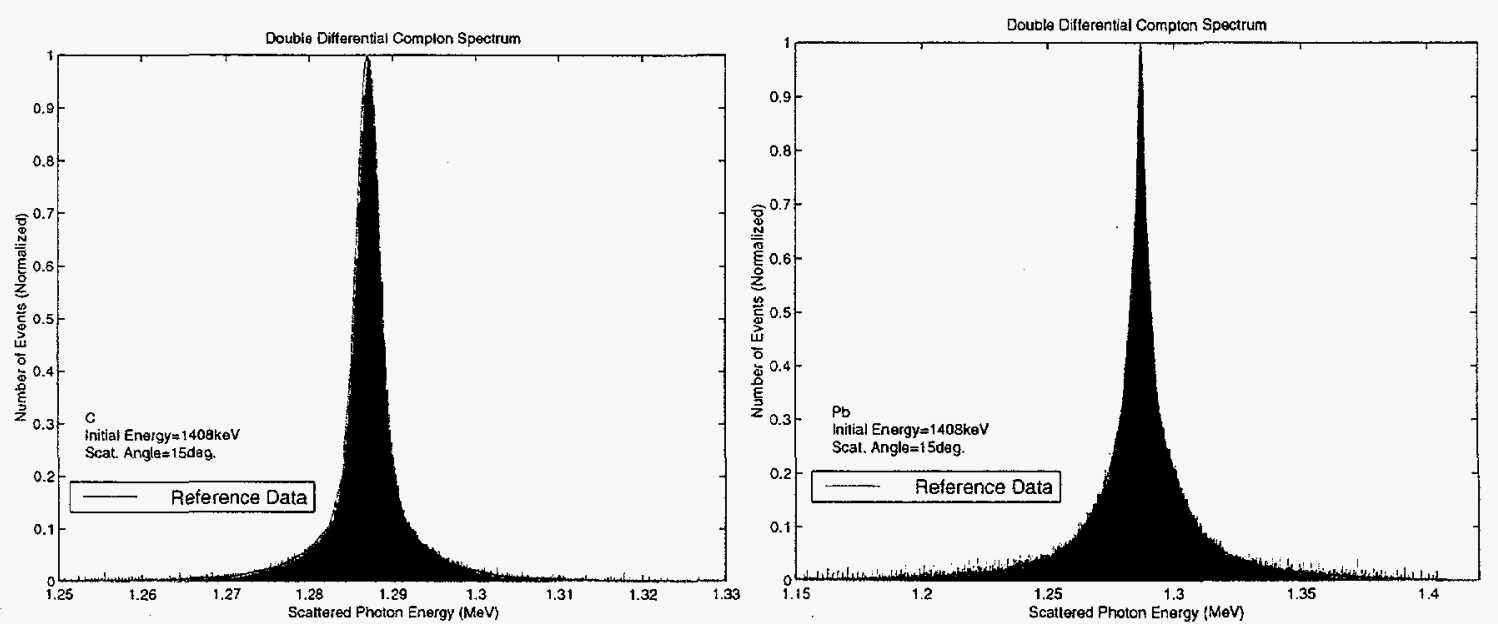

Fig. 4. Comparison of Monte Carlo simulation results (histogram) and theoretical calculations (red line) of the double differential Compton scattering profiles for carbon (left) and lead (right) for an initial photon energy of $1408 \mathrm{keV}$ and a scattering angle of 15 degrees.

Apparent in all of these figures is a shift in scattered photon energy between the theoretical calculations and Monte Carlo results. The magnitudes of the shifts are $96 \mathrm{eV}$, $93 \mathrm{eV}, 191 \mathrm{eV}$, and $163 \mathrm{eV}$ for Figs. 1-4, respectively. The shifts are less than $0.03 \%$ of the peak energy in all cases. The peak in the Monte Carlo results is always at the freeelectron scatter energy calculated by the Compton energy-angle relation (corresponding to the case when the determinate of the solution of the momentum equation is zero). The derivation of the momentum equation is based on energy-momentum conservation assuming free particle kinematics. Therefore, the energy peak from these routines will always be at the free-electron scatter energy. The relativistic impulse approximation solutions do not contain any assumptions regarding the energy value of the peak. 
The single differential Compton scattering cross section was calculated by numerical integration of the double differential cross section (from the combined output of the subroutines, kahnd.for and doppler.for) over the scattered photon energy. The double differential cross section was divided into 1000 energy bins and 360 polar angle bins for $10^{7}$ histories. The energy range covered was $\frac{0.01 E_{i}}{1+2 \alpha_{i}} \leq E_{s} \leq 1.1 E_{i}$, and polar angle range was $[0,2 \pi]$. The integral was properly normalized and compared with a direct calculation of the Klein-Nishina formula multiplied by the incoherent scattering function. These comparisons are presented in Figs. 5-7 for carbon and lead at initial photon energies of 10,344 , and $1408 \mathrm{keV}$.
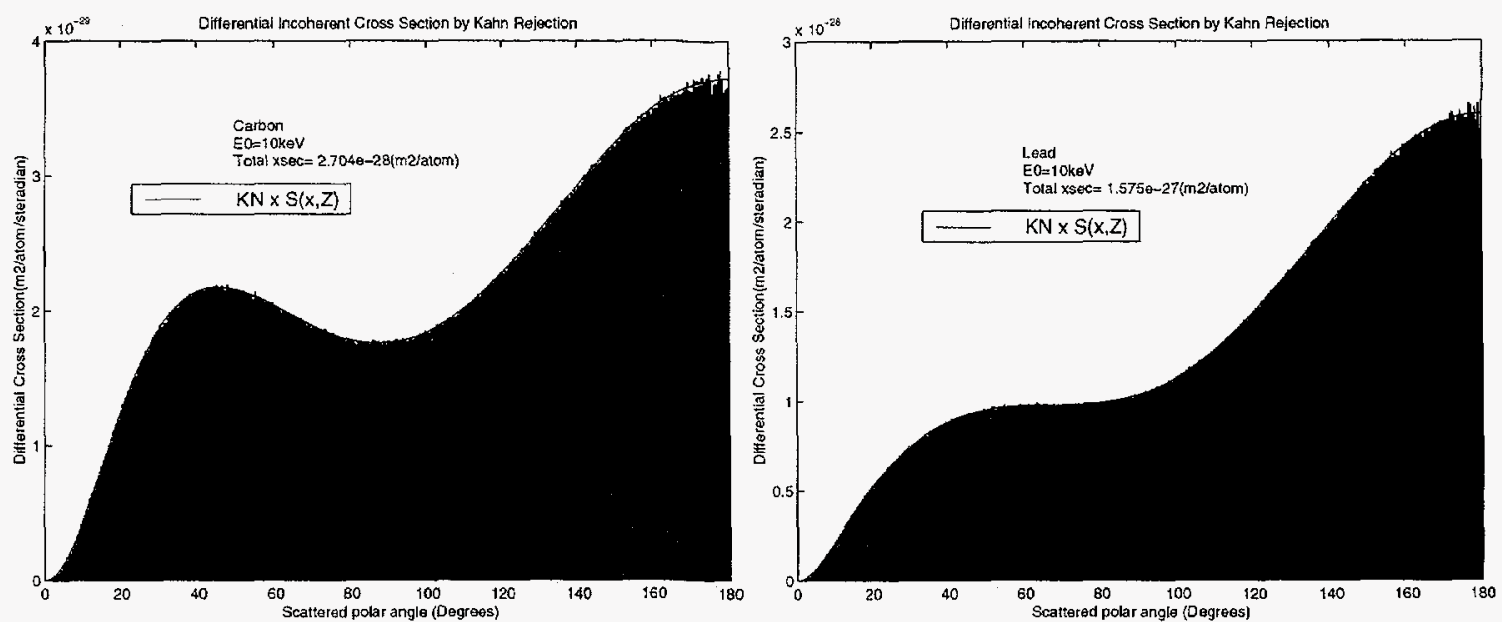

Fig. 5. Comparison of Monte Carlo simulation results (histogram) and direct calculations (red line) of the differential Compton scattering cross section for carbon (left) and lead (right) for an initial photon energy of $10 \mathrm{keV}$.
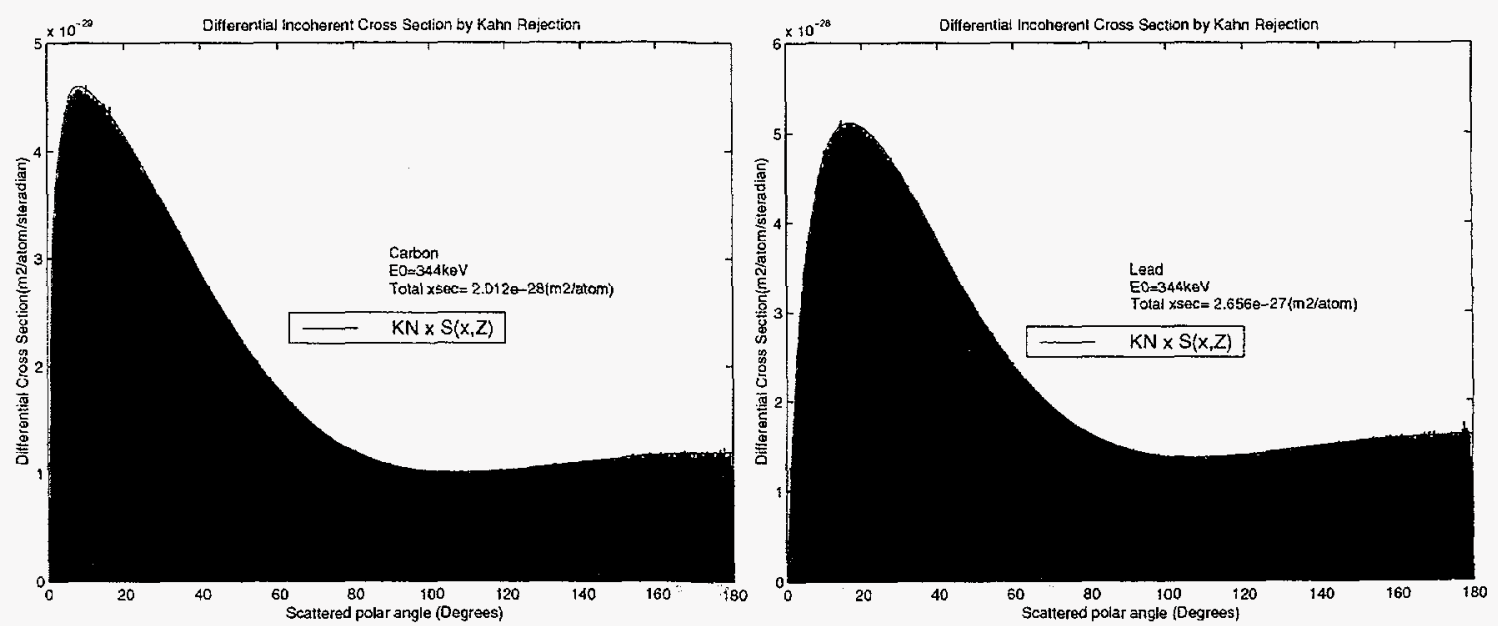

Fig. 6. Comparison of Monte Carlo simulation results (histogram) and direct calculations (red line) of the differential Compton scattering cross section for carbon (left) and lead (right) for an initial photon energy of $344 \mathrm{keV}$. 

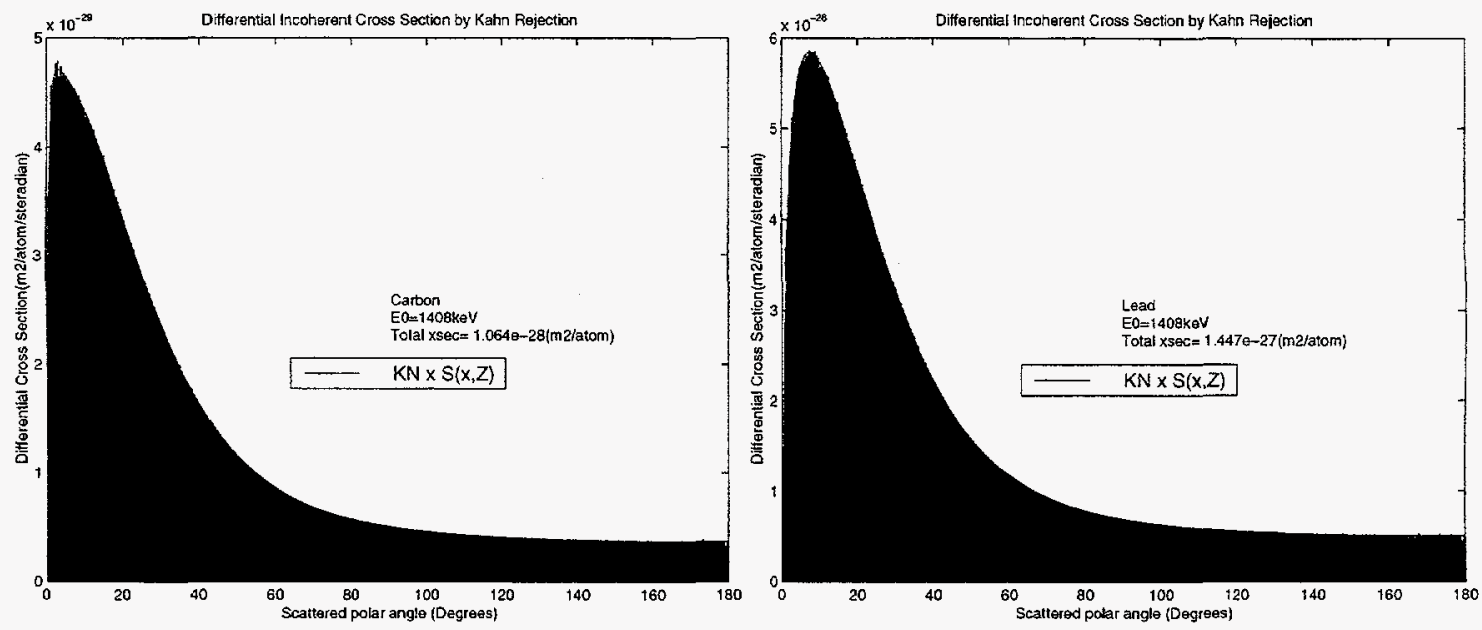

Fig. 7. Comparison of Monte Carlo simulation results (histogram) and direct calculations (red line) of the differential Compton scattering cross section for carbon (left) and lead (right) for an initial photon energy of $1408 \mathrm{keV}$.

In order to verify the calculations over $1 \leq Z \leq 100$ and $0.01 \mathrm{keV} \leq E_{i} \leq 100 \mathrm{meV}$, total Compton scattering cross sections were calculated and compared with values from the Evaluated Photon Data Library maintained at Lawrence Livermore National Laboratory [2]. The total cross sections were calculated by numerical integration of the single differential cross sections over the full solid angle. Figure 8 contains the specific comparisons for all $\mathrm{Z}$ and initial photon energies of $0.01 \mathrm{keV}, 0.1 \mathrm{keV}, 1 \mathrm{MeV}, 10 \mathrm{MeV}$, and $100 \mathrm{MeV}$. The largest error was $1.08 \%$ different from the published value. The subroutines are capable of calculations below $10 \mathrm{keV}$, although runtimes may become excessive due to reduced efficiency of the incoherent scattering function rejection technique.

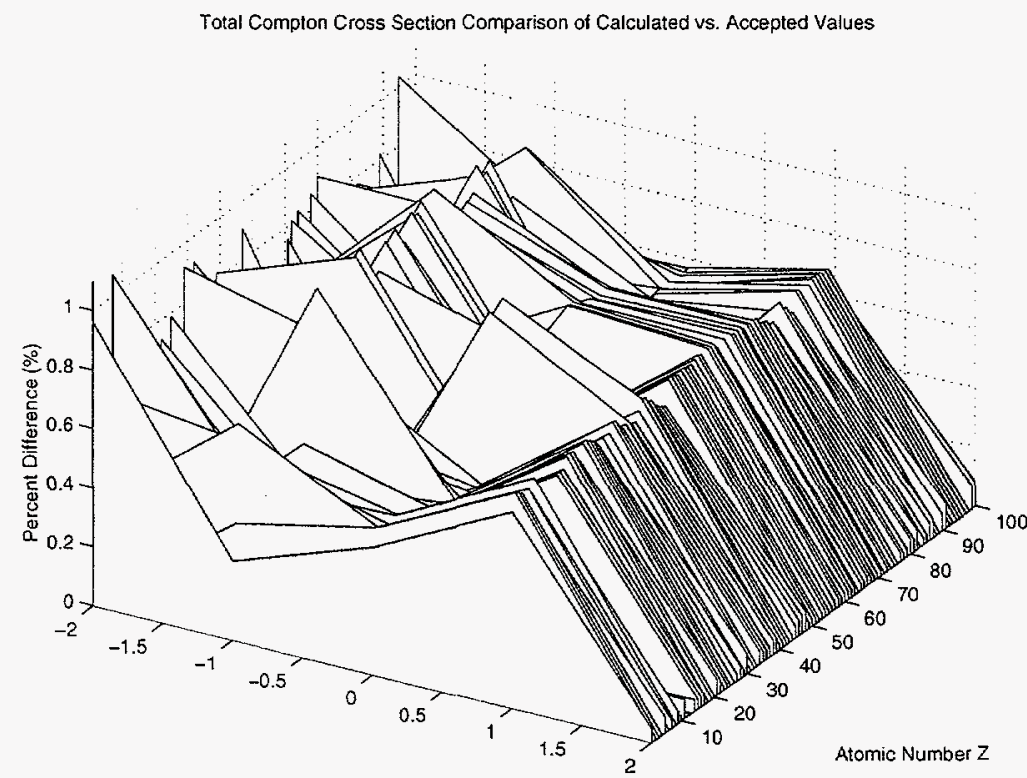

Log of Photon Energy (MeV)

Fig. 8. Total Compton cross section comparison between calculated values and accepted values. 


\section{CONCLUSIONS}

Two Monte Carlo subroutines have been coded to treat the physics of bound electron Compton scattering for unpolarized photons. The range of input parameters for which quality assurance has been demonstrated are $1 \leq Z \leq 100$ and $0.01 \mathrm{keV} \geq E_{i} \geq 100 \mathrm{MeV}$. The subroutines provide the Compton-scattered photon energy, polar angle, and azimuthal angle (both referenced from the original direction of the initial photon) as output.

\section{ACKNOWLEDGEMENTS}

The authors express thanks to Robert Little of the X-CI group at Los Alamos National Laboratory for providing the incoherent scattering function and total incoherent scattering cross section data.

\section{REFERENCES}

[1] H. Kahn, "Applications of Monte Carlo," AEC-3259, The Rand Corporation (1956).

[2] D.E. Cullin, M.H. Chen, J.H. Hubbell, et al., "Tables and graphs of photon interaction cross sections from $10 \mathrm{eV}$ to $100 \mathrm{GeV}$ derived from the LLNL Evaluated Nuclear Data Library (ENDL)," UCRL-50400, Vol. 6, Rev. 4, Part A: $\mathrm{Z}=1-50$ and Part B: $\mathrm{Z}=51-100$, Lawrence Livermore National Laboratory (1989).

[3] B. Williams, ed., Compton Scattering: The Investigation of Electron Momentum Distributions (Mcgraw-Hill New York, 1977).

[4] F. Biggs, L.B. Mendelsohn, J.B. Mann, "Hartree-Fock Compton profiles for the elements," Atomic Data and Nuclear Data Tables, Vol. 16, pp. 201-309 (1975).

[5] Table of Isotopes, $8^{\text {th }}$ Edition, (John Wiley and Sons, Inc., New York, 1996) pp. F37-F39.

[6] Y. Namito, S. Ban, and H. Hirayama, "Implementation of the Doppler broadening of a Compton scattered photon into the EGS4 code," Nuclear Instruments and Methods in Physics Research, A349, pp.489-494 (1994).

[7] J.C. Dow, J. P.Lestone, I.B. Whittingham, "Relativistic impulse approximation calculations of Compton scattering of Eu152 and Eu154 gamma rays," Natural Philosophy Research Report No. 82, Physics Dept., James Cook University of North Queensland, Australia (1986).

[8] L.L. Carter and E.D. Cashwell, Particle Transport Simulation with the Monte Carlo Method, Los Alamos National Laboratory, TID-26607 (1975). 


\section{APPENDIX A - Incoherent Scattering Function Rejection Technique}

In order to account for the interference effects from the nucleus and other electrons around the atom, the Klein-Nishina differential cross section, $f\left(\alpha_{i}, \mu\right) d \mu$, is modified by multiplication of the incoherent scattering function

$$
\sigma^{i n c}\left(\alpha_{i}, Z, \mu\right) d \mu=S\left(x\left(\mu, \alpha_{i}\right), Z\right) f\left(\alpha_{i}, \mu\right) d \mu \quad .
$$

The probability distribution function (pdf) for incoherent scattering is

$$
p\left(\alpha_{i}, Z, \mu\right)=\frac{\sigma^{i n c}\left(\alpha_{i}, Z, \mu\right)}{\int_{-1}^{1} \sigma^{i n c}\left(\alpha_{i}, Z, \mu\right) d \mu} .
$$

This can be written in the form

$$
p\left(\alpha_{i}, Z, \mu\right)=\frac{\sigma^{i n c}\left(\alpha_{i}, Z, \mu\right)}{\int_{-1}^{1} \sigma^{i n c}\left(\alpha_{i}, Z, \mu\right) d \mu} \times \frac{S\left(x\left(-1, \alpha_{i}\right), Z\right)}{S\left(x\left(-1, \alpha_{i}\right), Z\right)} \times \frac{\int_{-1}^{1} f\left(\alpha_{i}, \mu\right) d \mu}{\int_{-1}^{1} f\left(\alpha_{i}, \mu\right) d \mu},
$$

and by rearranging terms,

$$
p\left(\alpha_{i}, Z, \mu\right)=\frac{S\left(x\left(-1, \alpha_{i}\right), Z\right) \int_{-1}^{1} f\left(\alpha_{i}, \mu\right) d \mu}{\int_{-1}^{1} S\left(x\left(\mu, \alpha_{i}\right), Z\right) f\left(\alpha_{i}, \mu\right) d \mu} \times \frac{S\left(x\left(\mu, \alpha_{i}\right), Z\right)}{S\left(x\left(-1, \alpha_{i}\right), Z\right)} \times \frac{f\left(\alpha_{i}, \mu\right)}{\int_{-1}^{1} f\left(\alpha_{i}, \mu\right) d \mu}
$$

now,

$$
p\left(\alpha_{i}, Z, \mu\right)=C_{0} g\left(\mu, \alpha_{i}, Z\right) h\left(\alpha_{i}, \mu\right)
$$

A generalized rejection technique can be applied to a probability distribution function of this form, where $C_{0}$ is a normalization constant, $0 \leq g\left(\mu, \alpha_{i}, Z\right) \leq 1$ and $h\left(\alpha_{i}, \mu\right)$ is a probability distribution function bounded by the same range as $p\left(\alpha_{i}, Z, \mu\right)$ [8]. First, sample $\mu$ from $h\left(\alpha_{i}, \mu\right)$ (the Klein-Nishina distribution, in this case). Now sample a random number, $\xi$, from a uniform distribution contained on $[0,1]$. If $\xi$ is less than $g\left(\mu, \alpha_{i}, Z\right)$, then the value of $\mu$ is retained, if not, then the process is repeated. 


\section{APPENDIX B - Users' Manual}

The incoherent scattering function and Compton profile data are tabulated into separate data files for each element by atomic number $(1 \leq Z \leq 100)$. For example, hydrogen files are named s001.data and z001.data, respectively. A subroutine called incdata.for reads in the data from all these files and precomputes values needed in kahnd.for and doppler.for. The relevant information for all $\mathrm{Z}$ is stored in a COMMON block. In addition, the uniform random number generator is "warmed up" in this program. The subroutine has no arguments and should be called before kahnd.for and doppler.for are called.

The two main subroutines that perform the relevant calculations are kahnd.for and doppler.for. The subroutine kahnd.for is called first. The input parameters are the initial photon energy in $\mathrm{MeV}$ and the atomic number of the scattering element. The subroutine returns the value of the cosine of the polar scattering angle and the azimuthal scattering angle in radians. These two angles are referenced from the original photon direction. Now, the subroutine doppler.for is called. The input parameters are the cosine of the polar scattering angle and the atomic number of the scattering element. This subroutine returns the scattered photon energy in $\mathrm{MeV}$.

These two subroutines, kahnd.for and doppler.for, require the use of two other support subroutines. A uniform random number distributed on the interval $[0,1]$ is produced by a subroutine genrnu.for, and log-log interpolation is provided by logint.for.

These subroutines are written in standard Fortran 77 and can be obtained in electronic format (including the data files) from Tom Prettyman at Los Alamos National Laboratory. 


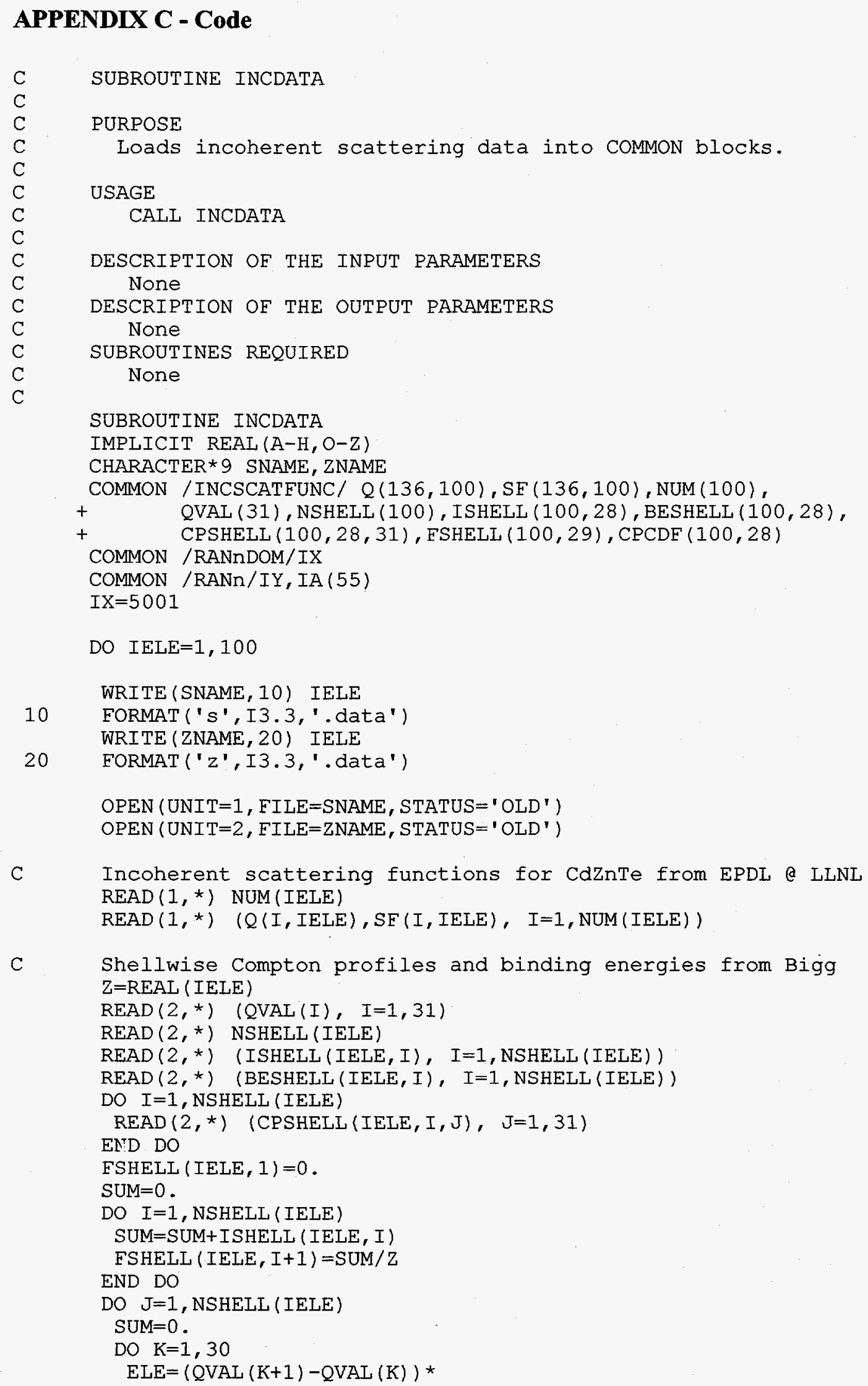


END DO

CPCDF (IELE, J) $=$ SUM

END DO

CLOSE (UNIT $=1$ )

CLOSE (UNIT $=2$ )

END DO

Prime the random number generator

CALL GENRNU

CALL RANNDM(RANN)

CALL RANNDM (RANN)

CALL RANNDM(RANN)

RETURN

END

C SUBROUTINE KAHND

C

C

\section{PURPOSE}

Determines the Compton scattered photon's angles using the Klein-Nishina probability distribution and the Kahn rejection technique. A rejection technique is used to include the electron's binding energy and momentum distribution around the nucleus via the total atom incoherent scattering function $S(x, z)$.

REFERENCES Herman Kahn, "Applications of Monte Carlo", Rand Corporation, RM-1237-AEC, 1956.

L.L. Carter and E.D. Cashwell, "Particle Transport Simulation with the Monte Carlo Method, Los Alamos Naional Laboratory, TID-26607, 1975.

USAGE

CALL KAHND (EI, IELE, AMU, PHI)

INPUT PARAMETERS:

EI - Photon's initial energy (MeV)

IELE -Atomic number of scattering element

\section{OUTPUT PARAMETERS:}

AMU -Cosine of the polar angle of scattered photon

PHI -Azimuthal angle of scattered photon (radians)

SUBROUTINES REQUIRED:

GENRNU.FOR -Uniform random number generator (MUST BE "WARMED UP" BY MAIN PROGRAM) .

LOGINT.FOR -Provides log-log interpolation of tabular values.

SUBROUTINE KAHND (EI, IELE, AMU, PHI)

IMPLICIT REAL (A-H, O-Z)

LOGICAL NOT DONE

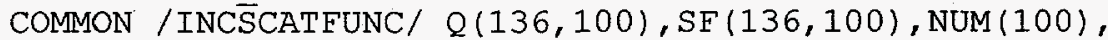

$+\quad$ QVAL (31), NSHELL (100), IShELL $(100,28), \operatorname{BEShELL}(100,28)$,

$+\quad \operatorname{CPSHELL}(100,28,31), \operatorname{FSHELL}(100,29), \operatorname{CPCDF}(100,28)$

COMMON /RANIDOM/IX 
$\mathrm{PI}=3.141592654$

EMASS $=0.511003101$

C Kahn rejection technique is used to determine the Compton

C scattered photon polar angle based on the Klein Nishina

C distribution

alp $=e i /$ emass

$\mathrm{x} 1=1$. +2 . *alp

NOT DONE $=$.TRUE.

DO W̄HILE (NOT DONE)

call ranndm( $\bar{r}$ ann 1 )

call ranndm(rann2)

call ranndm (rann3)

test $1=x 1 /(x 1+8$.

if (rann1.le.test 1$)$ then

$\mathrm{x}=1 .+\operatorname{rann} 2 *(\mathrm{x} 1-1$.

test $2=4 \cdot *(x-1) /.\left(x^{*} x\right)$

if (rann3. le.test2) then

NOT DONE $=$. FALSE.

endi $\bar{f}$

else

$x=x 1 /(1 .+\operatorname{rann} 2 *(x 1-1)$.

test $3=0.5 *(((a) p+1 .-x) / a l p) * * 2+1 . / x)$

if (rann3. le.test 3$)$ then

NOT DONE $=$. FALSE.

endi $\bar{f}$

endif

END DO

$a m u=1 .-(x-1.) / a l p$

if (abs (amu).gt.1.) amu=sign(1.,amu)

C Rejection technique to account for incoherent scattering function $\mathrm{QMAX}=29.14$ * $(\mathrm{EI} / \mathrm{EMASS})$ * $\mathrm{SQRT}(2$.

CALL LOGINT (NUM (IELE), $Q(1$, IELE), SF (1, IELE), QMAX, SMAX)

QVALUE $=29.14 *($ EI $/$ EMASS $) *$ SQRT $(1-$ AMU $)$

CALL LOGINT (NUM (IELE), Q (1, IELE), SF (1, IELE), QVALUE, SVAL)

CALL RANNDM (RANN)

IF (RANN* SMAX.GT.SVAL) GOTO 10

C

$\mathrm{C}$

Scattered photon azimuthal angle is determined assuming initial

photon is unpolarized

CALI RANNDM (RANN)

$\mathrm{PHI}=2 \star \mathrm{PI} \star \mathrm{RANN}$

RETURN

END

SUBROUTINE DOPPLER

PURPOSE

Calculates a Compton scattered photon's energy via doppler broadening.

\section{REFERENCE}

Y. Namito, S. Ban, H. Hirayama "Implementation of the Doppler Broadening of a Compton-Scattered Photon into the EGS4 code" Nuclear Instruments and Methods in Physics Research: A349, 
$C$
$C$
$C$
$C$
$C$
$C$
$C$
$C$
$C$
$C$
$C$
$C$
$C$
$C$
$C$
$C$
$C$
$C$
$C$

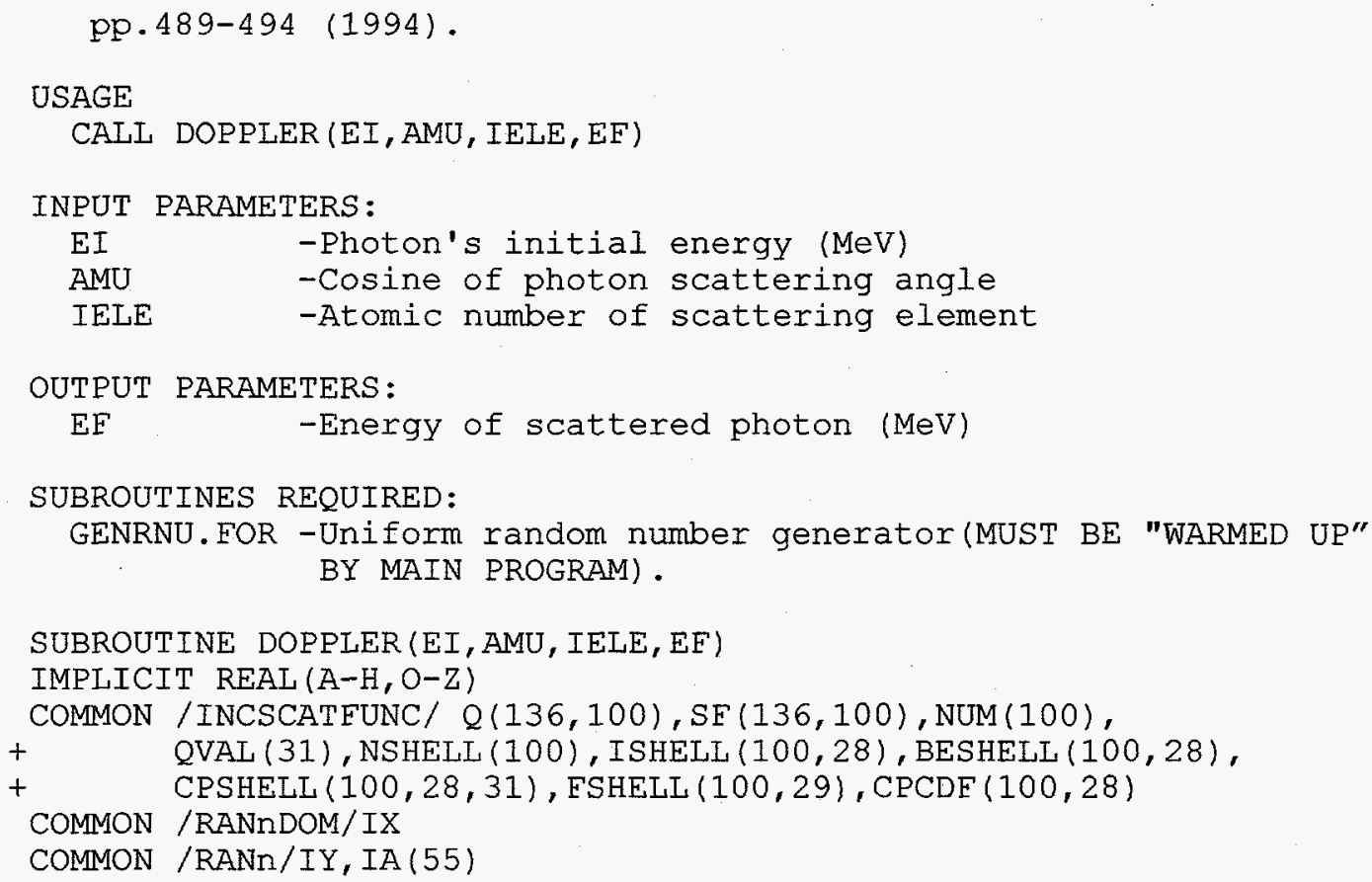




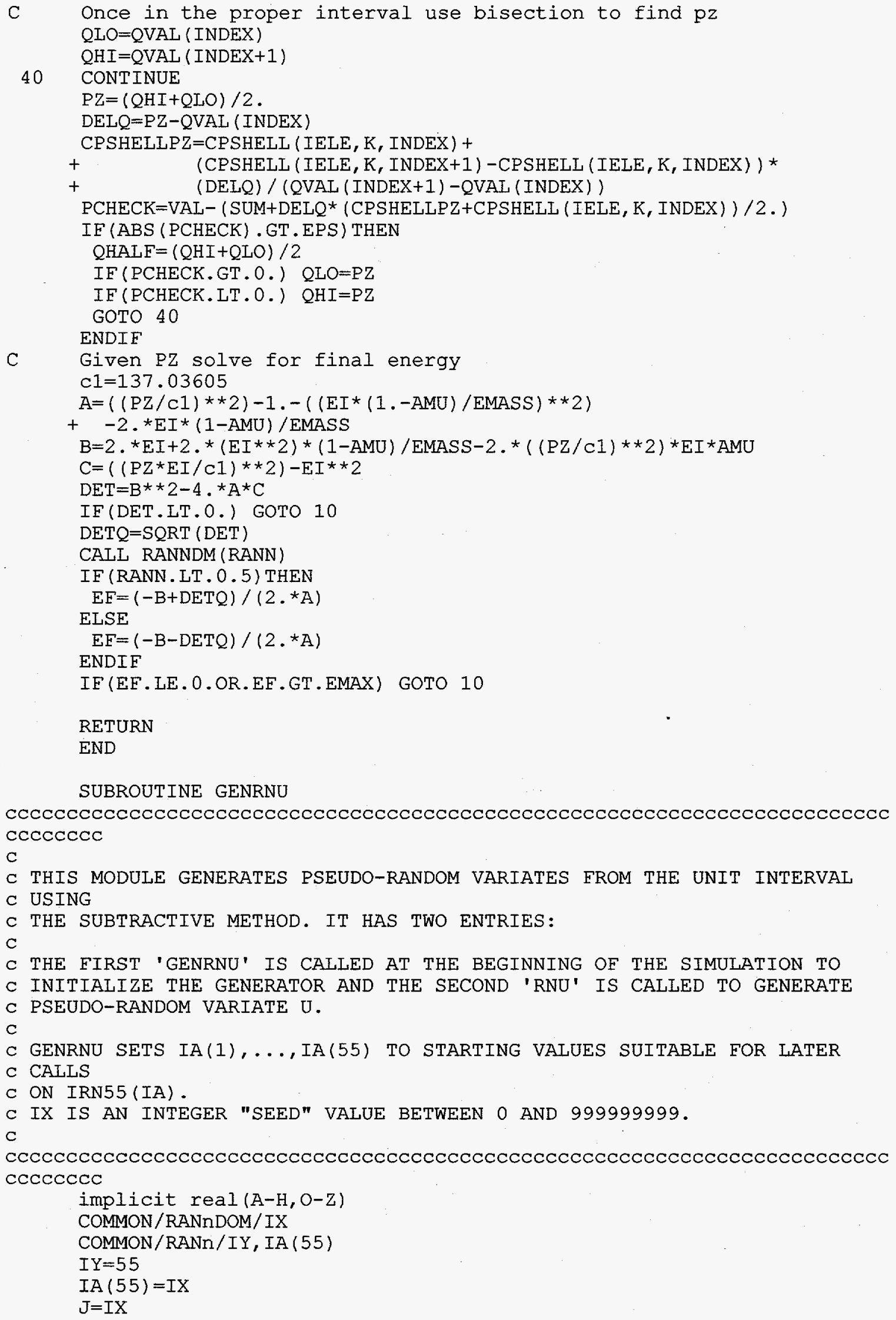




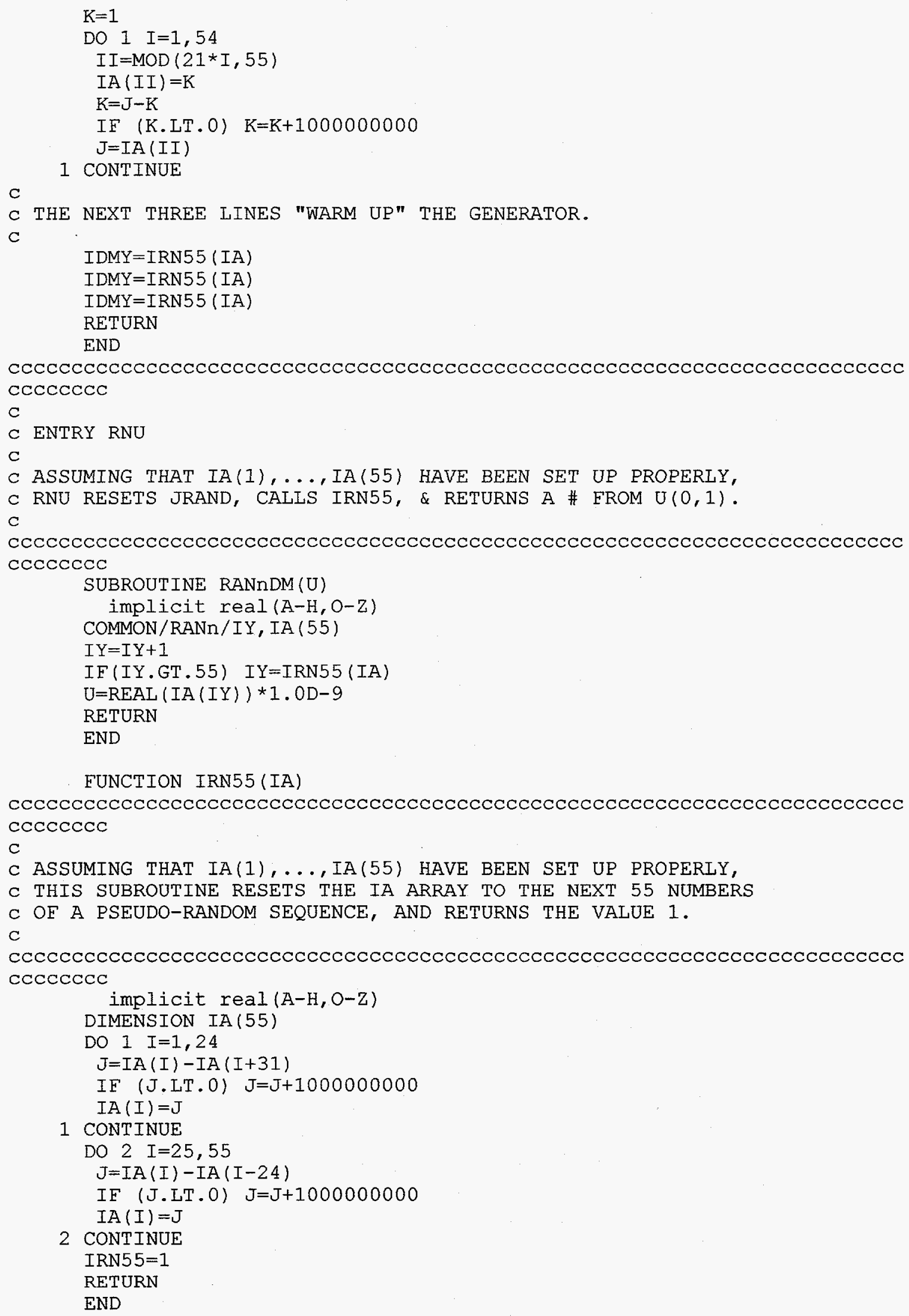


PURPOSE

Given arrays $X(I)$ and $Y(I)$ of length $N$ containing a tabulated function or data with minimal error, with $X(1)<X(2)<X(3) \ldots<x(n)$, a value of $X$ (XDUM) within the range of $X$ from $X(1)$ to $X(N)$, this subroutine returns a log-log interpolated value of $Y$ (YDUM).

USAGE

CALL LOGINT ( $N, X, Y, X D U M, Y D U M)$

INPUT PARAMETERS

$\mathrm{N}$ - Integer number of tabulated or data points.

$\mathrm{X}$ - Vector of data values for the independent variable.

$Y$ - Vector of data values for the dependent variable.

$X D U M-V a l u e$ of $X$ between $X(1)$ and $X(N)$.

OUTPUT PARAMETERS

YDUM- Value of $Y$ that matches XDUM obtained by log-log

interpolation.

SUBROUTINE LOGINT (N, X, Y, XDUM, YDUM)

IMPLICIT REAL $(\mathrm{A}-\mathrm{H}, \mathrm{O}-\mathrm{Z})$

DIMENSION $\mathrm{X}(150), \mathrm{Y}(150)$

Some constants are set.

$\mathrm{ZERO}=0.0$

The proper interval as denoted by the integer $\mathrm{K}$ is found by a bisection method.

$\mathrm{KLO}=1$

$\mathrm{KHI}=\mathrm{N}$

1 CONTINUE

$\mathrm{KCHECK}=\mathrm{KHI}-\mathrm{KLO}$

IF (KCHECK. GT . 1) THEN

$\mathrm{K}=(\mathrm{KHI}+\mathrm{KLO}) / 2$

IF (X (K) . GT . XDUM) KHI $=K$

IF (X (K) . LE. XDUM) KLO=K

GO TO 1

ENDIF

C

C

C

C

C

C

C

Now the KLO and KHI bracket the input $\mathrm{X}$ value of XDUM and differ by one.

The data points must be unique. (One could not use discontinuous functions in this routine.) This is checked in the following.

$\mathrm{H}=\mathrm{X}(\mathrm{KHI})-\mathrm{X}(\mathrm{KLO})$

IF (H. EQ. ZERO) THEN

WRITE $(*, *)$ 'Bad $X(I)$ input, $H$ is zero.'

RETURN

ENDIF

The LOG-LOG interpolation is done next (linear interpolation is done if the first point is at $x(k l o)=0)$.

IF (X (KLO) . GT . 0 . ) THEN

$X L O=L O G 10(X(K L O))$

$Y L O=L O G 10(Y(K L O))$ 


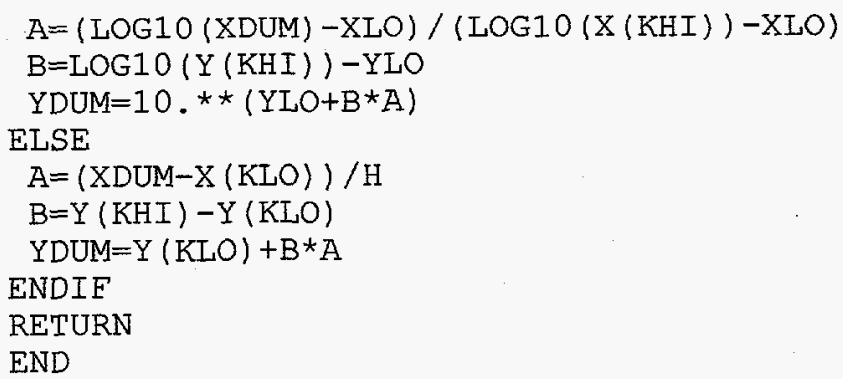

